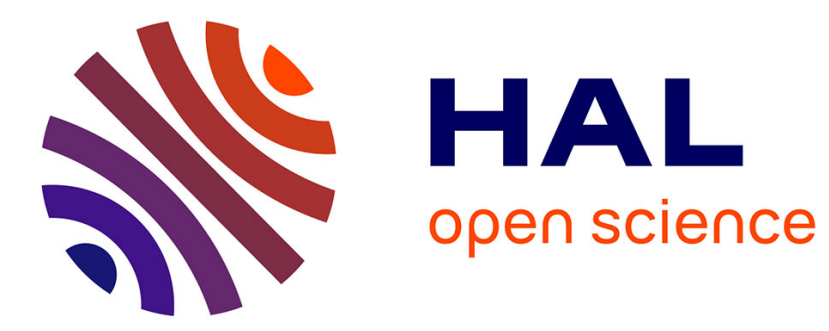

\title{
OPTICAL CONSTANTS AND THEIR MEASUREMENT
}

D. Lynch

\section{To cite this version:}

D. Lynch. OPTICAL CONSTANTS AND THEIR MEASUREMENT. Journal de Physique Colloques, 1977, 38 (C5), pp.C5-21-C5-30. 10.1051/jphyscol:1977503 . jpa-00217150

\section{HAL Id: jpa-00217150 https://hal.science/jpa-00217150}

Submitted on 1 Jan 1977

HAL is a multi-disciplinary open access archive for the deposit and dissemination of scientific research documents, whether they are published or not. The documents may come from teaching and research institutions in France or abroad, or from public or private research centers.
L'archive ouverte pluridisciplinaire HAL, est destinée au dépôt et à la diffusion de documents scientifiques de niveau recherche, publiés ou non, émanant des établissements d'enseignement et de recherche français ou étrangers, des laboratoires publics ou privés. 


\title{
OPTICAL CONSTANTS AND THEIR MEASUREMENT
}

\author{
D. W. LYNCH \\ Ames Laboratory-ERDA and Department of Physics, \\ lowa state University, Ames, Iowa, 50011 U. S. A.
}

\begin{abstract}
Résumé. - Nous exposons dans ses lignes générales la méthode de la fonction diélectrique et ses limites dans la description des propriétés optiques des solides, en particulier des métaux. Nous décrivons aussi des méthodes pour déduire cette fonction à partir des expériences. Nous soulignons l'importance d'erreurs dérivant de la préparation des échantillons plutôt que de la technique de mesure. On discute les données expérimentales pour différents éléments métalliques.
\end{abstract}

Abstract. - A description of the optical properties of solids, especially metals, in terms of a dielectric function is outlined, along with some of its limitations. Methods of deriving this function from measurements are outlined as well. Errors due to sample preparation, rather than measurement errors, are emphasized. Experimental data for several elemental metals is discussed.

1. Introduction. - We review the description of the optical properties of solids in terms of a dielectric function, indicating some of the limitations to such a description. Most of the limitations to be discussed are more likely to be encountered in metals than in semiconductors or insulators, and metals are of greater interest in photoelectrochemistry, so the subsequent discussion and examples will be limited to metals. We outline briefly a microscopic model for the dielectric function which illustrates some other general properties of dielectric functions (which, however, are modelindependent). We then outline general methods for obtaining dielectric functions from experimental data, discussing experimental errors, conceptual errors, and problems with sample preparation. Several sets of data will then be examined.

2. The dielectric function. - For a steady applied electric field the linear response of a homogeneous isotropic dielectric medium is given by a dielectric constant $\varepsilon$ defined by

$$
\mathbf{D}=\varepsilon \mathbf{E},
$$

while the linear response of a similarly simplified conductor is characterized by a conductivity $\sigma$, defined by

$$
\mathbf{J}=\sigma \mathbf{E} .
$$

If the electric field is time-dependent, the response generally will not be instantaneous, so that we must use a time-dependent conductivity and dielectric constant in the above constitutive equations. It is easier to Fourier analyze all time-dependent quantities and write

$$
\begin{aligned}
& D_{\omega} \mathrm{e}^{-i \omega t}=\varepsilon(\omega) E_{\omega} \mathrm{e}^{-i \omega t} \\
& J_{\omega} \mathrm{e}^{-i \omega t}=\sigma(\omega) E_{\omega} \mathrm{e}^{-i \omega t},
\end{aligned}
$$

which introduce the frequency-dependent dielectric constant and conductivity. The Fourier transforms of these, the time-dependent constitutive parameters, are rarely used. Both constitutive parameters, now to be called functions because of their frequency dependence, can be combined by substitution into one of Maxwell's equations :

$$
\left.\begin{array}{rl}
\nabla \times \mathbf{H} & =\frac{1}{c} \frac{\partial \mathbf{D}}{\partial t}+\frac{4 \pi}{c} \mathbf{J} \\
& =\frac{1}{c}[-i \omega \varepsilon(\omega)+4 \pi \sigma(\omega)] \mathbf{E}_{\omega} \mathrm{e}^{-i \omega t} \\
& =\frac{1}{c} 4 \pi \tilde{\sigma}(\omega) \mathbf{E}_{\omega} \mathrm{e}^{-i \omega t} \\
& =\frac{1}{c}\left[\varepsilon(\omega)+i \frac{4 \pi}{\omega} \sigma(\omega)\right] \frac{\partial}{\partial t}\left(\mathbf{E}_{\omega} \mathrm{e}^{-i \omega t}\right) \\
& =\frac{1}{c} \tilde{\varepsilon}(\omega) .
\end{array}\right\}
$$

These define [1-3] the complex conductivity $\tilde{\sigma}(\omega)$ and complex dielectric function $\tilde{\varepsilon}(\omega)$, which are related by

$$
\tilde{\sigma}(\omega)=-\frac{i \omega}{4 \pi} \tilde{\varepsilon}(\omega)
$$

The imaginary part of $\tilde{\varepsilon}(\omega)$ and the real part of $\tilde{\sigma}(\omega)$ describe dissipative processes, for

$$
\tilde{\varepsilon}(\omega)=\varepsilon_{1}(\omega)+i \varepsilon_{2}(\omega)=\varepsilon(\omega)+i \frac{4 \pi}{\omega} \sigma(\omega)
$$

and

$$
\tilde{\sigma}(\omega)=\sigma_{1}(\omega)+i \sigma_{2}(\omega)=\sigma(\omega)-i \frac{\omega \varepsilon(\omega)}{4 \pi} .
$$

In crystalline media the dielectric function becomes a symmetric Cartesian tensor which can be diagonalized by a suitable choice of axes. For cubic crystals, all three diagonal components are equal and tensor nota- 
tion is not needed. For hexagonal, trigonal, and orthorhombic crystals, the principal axes coincide with crystallographic axes, but in other systems not all axes coincide, giving, in effect, up to three directions and three (complex) diagonal components as the necessary quantities to characterize the optical response [4]. At optical frequencies, the magnetic response of matter is negligible, but an applied static field or permanent magnetization makes the dielectric function of all media tensorial.

If the applied electric field becomes large, the linear approximation breaks down and we enter the regime of nonlinear optics. By not using high power densities, this region can be avoided, and we do so here.

The most important remaining restriction on the dielectric function defined above is that it is local in space, although it is not local in time. Taking just the conductivity as an example, locality implies

$$
J_{\omega}(\mathbf{r})=\sigma(\omega) \mathbf{E}_{\omega}(\mathbf{r}) .
$$

If, for any reason, the charge carriers at $\mathbf{r}$ have velocities determined by electric fields at other points, $\mathbf{r}^{\prime}$ where the field is appreciably different from the field at $r$, one must write

$$
J_{\omega}(\mathbf{r})=\int \sigma\left(\omega, \mathbf{r}, \mathbf{r}^{\prime}\right) E_{\omega}\left(\mathbf{r}^{\prime}\right) \mathrm{d}^{3} \mathbf{r}^{\prime} .
$$

This is a nonlocal situation because the current density at $\mathbf{r}$ depends on the fields in a region around the point $\mathbf{r}$. Rather than deal with this integral, it is usual to Fourier analyze this constitutive function with respect to $\mathbf{r}^{\prime}$. If the material is homogeneous

$$
\sigma\left(\omega, \mathbf{r}, \mathbf{r}^{\prime}\right)=\sigma\left(\omega, \mathbf{r}-\mathbf{r}^{\prime}\right)
$$

and the Fourier transform depends only on the conjugate variable to $\mathbf{r}$, the wave vector $\mathbf{k}$, giving $\sigma(\omega, \mathbf{k})$. A medium exhibiting $\mathbf{k}$-dependence in its constitutive functions is said to exhibit spatial dispersion. There are a number of systems in which spatial dispersion is important. It is a prerequisite for optical activity. It occurs in insulators and semiconductors in the spectral region of exciton creation. In metals, the anomalous skin effect is a good example of a nonlocal situation [5, 6]. When the electron mean free path becomes longer than the classical skin depth, the current in the skin depth region is determined by the fields the electrons in that region have experienced over their previous path, back to their last collision. The current then depends on the electric field over a depth larger than the skin depth, a field which varies from its surface value to nearly zero, and an integral over the path must be performed. In fact, the anomalous skin effect, which is very large in the microwave and far infrared regions for pure metals at low temperatures, may cause small to appreciable changes in the optical properties in the near infrared, and even the visible, for free electron-like metals at room temperatures, especially if scattering from the surface is nonspecular [7]. In such a situation, it is misleading to try to use a simple frequency-dependent dielectric function. In fact, even for an isotropic medium, two dielectric functions are needed [2], a longitudinal and a transverse, for situations in which the field $\mathbf{E}$ is parallel or perpendicular to $\mathbf{k}$. Since optical measurements alone will not lead to $\tilde{\varepsilon}(\omega, \mathbf{k})$, and $\tilde{\varepsilon}(\omega)$ is misleading, one should report data in such situations in terms of the surface impedance, $\widetilde{Z}=(4 \pi / c)(\tilde{E} / \tilde{H})_{\text {surf }}$, or quantities derived from it, such as the absorptance or reflectance,

$$
R=|\widetilde{Z}-4 \pi / c|^{2} /|\widetilde{Z}+4 \pi / c|^{2}
$$

for normal incidence from vacuum $[2,6,8,9]$. The use of a local dielectric function in a nonlocal situation can lead to obvious problems if the measurements made lead to an overdetermined set of equations for finding the dielectric function, for acceptable solutions may not be found and there may be a large dependence on polarization. Without such an overdetermined set or a consistency check, the need for nonlocality may not be recognized.

Finally, one must take account of nonhomogeneity. All samples have at least one surface involved in a measurement. The outer layers, even if not oxidized or strained from preparation, may not be the same as the bulk. The surface atoms may be further from their neighbours, there may be microscopic puckering of the surface atoms, and the charge density of the valence conduction electrons will die out into the next medium with a length scale of the order of the Thomas-Fermi screening length, no more than $1 \AA$. One could ascribe a different dielectric function to a surface region $[10$, 11], but this increases the number of unknowns to be determined from experiment, and the concept of a dielectric function for a region no thicker than a unit cell is not a clear one. One could introduce a local $\tilde{\varepsilon}(\omega, \mathbf{r})$ or nonlocal $\tilde{\varepsilon}\left(\omega, \mathbf{r}, \mathbf{r}^{\prime}\right)[12]$, or treat the surface region microscopically $[13,14]$. At any rate, when an experimental method involves the use of $p$-polarized radiation at non-normal incidence, the details of the surface response become important, for it is what determines the distribution in depth of the induced « surface » charge density. If we knew the bulk dielectric functions well enough, such measurements could be used to probe the surface charge distribution. Photoemission measurements [15] also do so, but much remains to be done in this area. The role of the surface is especially important in the anomalous skin effect regime.

The dielectric function is linear and causal. Its real and imaginary parts obey dispersion or KramersKronig relations :

$$
\begin{gathered}
\varepsilon_{1}(\omega)-1=\frac{2}{\pi} P \int_{0}^{\infty} \frac{\varepsilon_{2}\left(\omega^{\prime}\right) \omega^{\prime} \mathrm{d} \omega^{\prime}}{\omega^{\prime 2}-\omega^{2}} \\
\varepsilon_{2}(\omega)-\frac{4 \pi \sigma_{0}}{\omega}=\frac{-2 \omega}{\pi} P \int_{0}^{\infty} \frac{\varepsilon_{1}\left(\omega^{\prime}\right) \mathrm{d} \omega^{\prime}}{\omega^{\prime 2}-\omega^{2}}
\end{gathered}
$$


in which $\sigma_{0}$ is the conductivity at zero frequency and the $P$ denotes principal value $[1,2]$. From these, one can obtain sum rules :

$$
\begin{aligned}
\int_{0}^{\infty} \omega \varepsilon_{2}(\omega) \mathrm{d} \omega & =\frac{\pi}{2} \omega_{\mathrm{p}}^{2} \\
\int_{0}^{\infty} \frac{\varepsilon_{2}(\omega) \mathrm{d} \omega}{\omega} & =\frac{\pi}{2}\left(\varepsilon_{1}(0)-1\right),
\end{aligned}
$$

in which the plasma frequency $\omega_{\mathrm{p}}=\left(4 \pi N e^{2} / \mathrm{m}\right)^{1 / 2}$, with $N$ the number density of electrons. These, and other sum rules $[1,2,16-18]$, are very useful for interpreting data and checking the consistency of data.

The derivation of the wave equation from Maxwell's equations leads to a complex refractive index $\tilde{N}$ related to the dielectric function by

$$
\left.\begin{array}{rl}
\tilde{N}^{2}=(n+i k)^{2}=\tilde{\varepsilon} \\
\varepsilon_{1} & =n^{2}-k^{2} \\
\varepsilon_{2} & =2 n k \\
2 n^{2} & =\left(\varepsilon_{1}^{2}+\varepsilon_{2}^{2}\right)^{1 / 2}+\varepsilon_{1} \\
2 k^{2} & =\left(\varepsilon_{1}^{2}+\varepsilon_{2}^{2}\right)^{1 / 2}-\varepsilon_{1}
\end{array}\right\} .
$$

Needless to say, these are valid at each frequency, but not when anisotropy or spatial dispersion must be considered. (The classical skin depth $\delta=c / \omega k$.)

For a planar, infinitely sharp interface between two media, $a$ and $b$, the boundary conditions derived from Maxwell's equations lead to the Fresnel relations for the ratio of the reflected and transmitted electric fields to the incident (from medium a) field [19] :

$$
\tilde{r}=\frac{\tilde{g}_{b}-\tilde{g}_{a}}{\tilde{g}_{b}+\tilde{g}_{a}}=r \mathrm{e}^{i \theta}, \quad \tilde{t}=1-\tilde{r}
$$

with

$$
\left.\begin{array}{l}
\tilde{g}_{\mathrm{i}}=\tilde{N}_{\mathrm{i}} \cos \tilde{\varphi}_{\mathrm{i}} \text { for s-polarization } \\
\tilde{\mathrm{g}}_{\mathrm{i}}=\left(\cos \varphi_{\mathrm{i}}\right) / \tilde{N}_{\mathrm{i}} \text { for p-polarization }
\end{array}\right\}
$$

and

$$
\tilde{N}_{\mathrm{a}} \sin \tilde{\varphi}_{\mathrm{a}}=\tilde{N}_{\mathrm{b}} \sin \tilde{\varphi}_{\mathrm{b}}
$$

(Usually medium a is nonabsorbing, so the angle of incidence, $\tilde{\varphi}_{\mathrm{a}}$ is real.) When the ambient medium, a, is air or vacuum, $\tilde{N}_{\mathrm{a}}=1$, and $\tilde{r}$ simplifies. Moreover, if then $\varphi_{\mathrm{a}}=45^{\circ},\left|\tilde{r}_{\mathrm{p}}\right|^{2}=\left|\tilde{r}_{\mathrm{s}}\right|^{4}$ for any $\tilde{N}_{\mathrm{b}}$. This, too can be used as a check on data, or on incomplete polarization. These can be generalized to a series of interfaces, considering interference effects if necessary $[11,19]$.

The reflection coefficient $\tilde{r}$ can be used in a dispersion relation, but since its real and imaginary parts each involve both $r$ and $\theta$, it is not a useful relation. However, $\ln \tilde{r}=\ln r+i \theta$ gives a very useful Kramers-Kronig pair, albeit a controversial one [20-24]. Despite the controversy, it remains a useful relationship, and one rarely encounters trouble when using it properly on a single interface.
The incident and reflected fields cannot be measured at optical frequencies; only their absolute squares can be measured. Thus, instead of $\tilde{r}$, one measures $R=|\tilde{r}|^{2}$, the power or intensity reflection coefficient, also called the reflectance or reflectivity. This is a function of frequency, angle of incidence, and polarization. Ellipsometric measurements give the ratio of two complex amplitude reflection coefficients, one for each polarization :

$$
\tilde{r}_{\mathrm{p}} / \tilde{r}_{\mathrm{s}}=\rho \mathrm{e}^{i \Delta}=(\tan \psi) \mathrm{e}^{i \Delta} .
$$

A sharp interface implies a transition from one medium to another in a distance that is small on some length scale. This is usually taken to be the wavelength of the measuring radiation or the optical penetration depth, whichever is smaller. For metals, it is usually the latter, the inverse of the absorption coefficient, $100 \AA$, or so. If we model the transition region between vacuum and a medium of dielectric function $\tilde{\varepsilon}_{\mathrm{b}}$ by introducing a thin region of thickness $d$ and dielectric function $\tilde{\varepsilon}_{0}$, then at normal incidence, if $d / \lambda$ is small ( $\lambda$ is the vacuum wavelength) the fractional change in reflectance is [11].

$$
\frac{\Delta R}{R} \approx-\frac{8 \pi d}{\lambda} \operatorname{Im}\left(\frac{\tilde{\varepsilon_{\mathrm{b}}}-\tilde{\varepsilon}_{0}}{\tilde{\varepsilon_{\mathrm{b}}}-1}\right) .
$$

If the last factor is of order unity, a $2 \AA$ thick transition region leads to a decrease in the reflectance of about 1 percent of the value of the reflectance. (This expression is inadequate if neither material is absorbing.)

The interface may be further complicated by an overlayer that is not continuous, or by a rough surface. In a number of cases, attempts to anneal the surface to remove damage from surface preparation leads to increased surface roughness [25], both representing departures from the mathematical interface supposedly under study. It is entirely possible that the surface roughness be on a wavelength scale, leading to resonant scattering effects [26-31]. Finally, in alloys, the surface composition may not be the same as the bulk composition.

A microscopic interpretation of the dielectric function is obtained by calculating the response of the microscopic model for the system to an applied electric field, which gives the resultant polarization or current [1]. A useful classical model, due to Lorentz, is that of bound damped charged harmonic oscillators [32]. There are $f_{i} N_{i}$ oscillators per unit volume each with mass $m$ charge $e$, resonant frequency $\omega_{l}$, and damping parameter $\Gamma_{i}$. The conduction electrons can be included by setting $\omega_{i}$ equal to zero. Optically active phonons can be included by using different charges and masses. Such a dielectric function is of the form

$$
\tilde{\varepsilon}(\omega)=1+\sum_{i} \frac{\left(4 \pi N_{i} f_{i} e^{2} / m\right)}{\omega_{i}^{2}-\omega^{2}-i \omega \Gamma_{i}} .
$$

The parameters $f_{i}, \omega_{i}$, and $\Gamma_{i}$ are fitted to the data 
and have no microscopic significance, except for the damping parameter in the conduction electron, or Drude, term, which is related to the static conductivity. Quantum mechanically one goes through a similar conceptual procedure, obtaining a current or polarization in response to the applied field. The conduction electron contribution can be found from a Boltzmann equation. The Drude terms may resemble the classical result in certain frequency regions, although the calculations have not been carried out for metals other than simple ones [33]. The harmonic oscillator contributions to $\tilde{\varepsilon}$ are replaced by a sum of integrals representing interband excitation of electrons [2]. The difficulties increase as one tries to treat more realistic models.

In a few simpler cases the calculation of the interband contribution to the dielectric function is quite reliable, e. g., in $\mathrm{Al}$ and possibly $\mathrm{Cu}$ and $\mathrm{Ag}$. Usually the momentum or electric dipole matrix elements cannot be calculated very accurately because of their great sensitivity to the wave functions. Unfortunately, the often-made approximation that the dipole matrix element is frequency-independent is not good enough, so in such cases we cannot use a calculated $\tilde{\varepsilon}$ as more than a rough guide to the measured $\tilde{\varepsilon}$. Certainly we cannot use theoretical dielectric functions to assess errors in measured dielectric functions, and only rarely can we use them to help distinguish the best measured data from among several disagreeing spectra. For disordered alloys, there have been no attempts to calculate a dielectric function, except with the rigid band model.

Structure in $\tilde{\varepsilon}$ in a limited range of frequency may arise from a group of similar transitions in the solid. In a metal, these may be transitions at a critical point or between parallel bands [34]. The observed structure in the entire spectrum may arise from several groups of such transitions. If we approximate each group of transitions by a single oscillator, we see from eq. (24), that the contributions of each group to $\tilde{\varepsilon}$ are additive. In $\varepsilon_{2}$ there is little overlap if the resonances are well separated, but in $\varepsilon_{1}$ the groups of electrons with high frequency resonances contribute a constant background at lower frequencies, even when the resonances are widely separated. This is illustrated in figure 1 for two oscillators. From $\tilde{\varepsilon}$, one can obtain $\tilde{N}$, then $R$. The contributions of each oscillator to the reflectance are not additive, however, and, although there may be a reflectance peak from each oscillator, one cannot fit the peaks separately to a dielectric function because of the contribution of higher frequency oscillators in the frequency range of all lower frequency oscillators. This is demonstrated in figure 1.

3. Experimental methods. - We now discuss several general methods for obtaining dielectric functions experimentally. We omit methods that make use of the high collimation of laser light, for usually these cannot give a
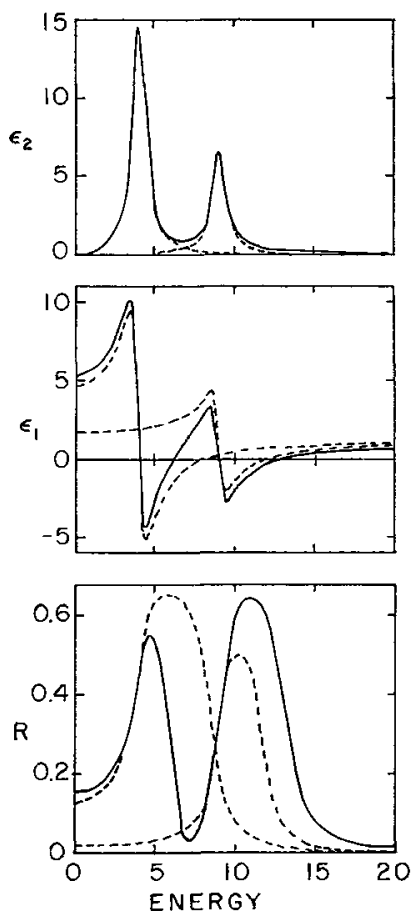

Frg. 1. - Real and imaginary parts of the dielectric function for two harmonic oscillators. The dashed lines represent these functions for each oscillator separately, and the solid line the sum of the two. The bottom curves are the reflectance of each oscillator alone (dashed) and of the two together (solid), which is not the sum of the two dashed curves.

continuous $\tilde{\varepsilon}(\omega)$ spectrum. Dye lasers are changing this, however.

3.1 Ellipsometry [35] will be discussed in detail in other papers at this conference. The accuracy and precision that can be obtained with ellipsometry can be very high, but it is not often achieved. The principal problems are alignment of the ellipsometer, the performance of its components, and the sensitivity of ellipsometry to surface films on the sample, even transparent films of less than a complete monolayer. Ellipsometry is, however, less sensitive than many other methods to surface roughness, for it is the polarization of the reflected radiation that is measured, not its absolute intensity. Ellipsometry becomes difficult in the infrared and vacuum ultraviolet [36]. In the infrared, the sensitivity falls, but it is often increased by using several samples or several reflections from a large sample. The lack of vacuum ultraviolet ellipsometric data is often not a serious problem for the study of the liquid-solid interface because of the opacity of nearly all liquids in the vacuum ultraviolet. A recent advance in ellipsometry is the development of automatic ellipsometers which, with a minicomputer, provide complete dielectric function spectra.

Another form of ellipsometry is polarization modulation [37-42]. Here a beam of radiation passes through a transparent plate which is driven to vibrate mechanically. The strain-induced birefringence in the plate 
periodically modulates the state of polarization of the radiation beam. The beam is then reflected from the sample which produces an additional change in the state of polarization. By synchronously detecting this beam at the fundamental and the second harmonic, one can obtain simple functions of the ellipsometric parameters of the sample, from which the dielectric function can be obtained.

3.2 There are many photometric measurements in which two reflected intensities are measured. The Fresnel equations are then solved numerically to yield $n$ and $k$ or $\varepsilon_{1}$ and $\varepsilon_{2}$. Measured quantities considered are

(1) $R\left(\varphi_{1}\right)$ and $R\left(\varphi_{2}\right)$ using unpolarized radiation [43].

(2) $\boldsymbol{R}_{\mathrm{p}}\left(\varphi_{1}\right)$ and $\boldsymbol{R}_{\mathrm{p}}\left(\varphi_{2}\right)[44,45]$.

(3) $R_{\mathrm{s}}\left(\varphi_{1}\right)$ and $R_{\mathrm{s}}\left(\varphi_{2}\right)[44,45]$.

(4) $R_{\mathrm{s}}\left(\varphi_{1}\right)$ and $R_{\mathrm{p}}\left(\varphi_{1}\right)[46,47]$.

(5) $R_{\mathrm{s}}\left(\varphi_{1}\right) / R_{\mathrm{p}}\left(\varphi_{1}\right)$ and $\boldsymbol{R}_{\mathrm{s}}\left(\varphi_{2}\right) / R_{\mathrm{p}}\left(\varphi_{2}\right)[48,44,45,50]$.

Others are possible. One can calculate the measured quantities from assumed values of $n$ and $k$ and obtain a plot of the measured quantities with the optical constants as parameters [51]. From these, the accuracy of the method can be assessed by assuming experimental errors in the measured quantities, and using the plots to determine the resultant errors in $n$ and $k$. Figure 2 illustrates such a plot for method (5). From

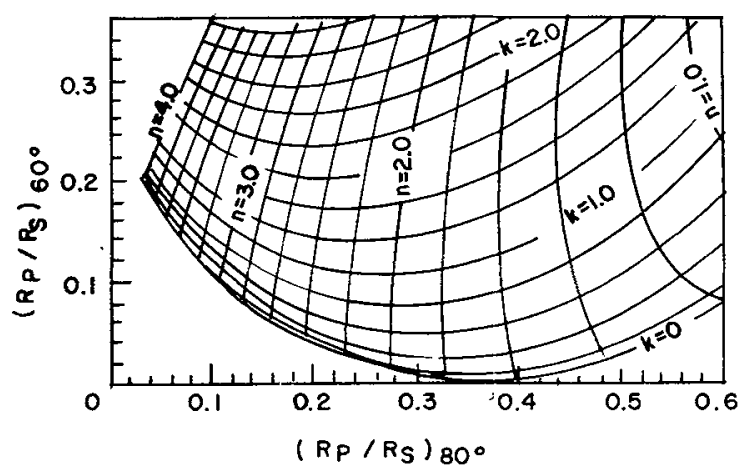

FIG. 2. - Curves of constant $n$ and $k$ as a function of two measured quantities, the $p$ - to s reflectance ratios at $60^{\circ}$ and $80^{\circ}$ angle of incidence [51].

this plot it can be seen that errors of \pm 0.01 in each of the measured quantities lead to errors of \pm 0.05 and $\stackrel{ \pm}{\sim} 0.07$ to $\approx 0.05$, respectively, in $n$ and $k$, for $n=2$ and $k=1$. These plots can be made for a variety of angles $\varphi$ in order to maximize the sensitivity for a given range of $n$ and $k$ values. No universal best method or best pair of $\varphi$ values exists. The first method is always difficult to use, for it requires completely unpolarized radiation. All of the methods are poor when $k$ is less than about 0.5 . When $n$ is small, say $\lesssim 1$, and $k$ is large, $>1$, as for a metal in the infrared, all the methods are poor unless $\varphi_{2}$ can be made large, $>70^{\circ}$ or even $80^{\circ}$. Method (3) is the least useful of the group, for it is particularly insensitive.
It is usually difficult to estimate the errors in measured photometric quantities. (See ref. [52] for an extensive discussion.) Rarely are such errors due to counting statistics. One must consider the degree of polarization actually achieved, the collimation of the measuring beam, the fraction of the reflected radiation actually collected, the fact that the sensitivity of the photocathode is a function of the position on the cathode, a function which may depend on the wavelength and polarization as well. (This problem may be diminished by using a diffusing screen in front of the detector but with appreciable reduction in signal.) Moreover, if two angles are used, the measuring beam samples different areas of the sample, an especially acute problem if a large angle of incidence is needed. Even if the experimenter can control these errors, it is difficult to convey to the reader of a paper the degree of control and its consequences.

Some improvement may be obtained by making more measurements, obtaining an overdetermined set of equations and solving them by a least squares fit to $n$ and $k$, with suitable weights assigned to the different measurements. Although this sounds better than two measurements, it is not clear that when this has been carried out, the results are more accurate.

3.3 There are a variety of other measurements one could make photometrically, some of which are measurements of an angle of incidence at which the reflectance for p-polarized radiation is a minimum. Call this angle $\varphi_{\mathrm{B}}$. Measurements considered are

(1) $\varphi_{\mathrm{B}}$ and $R_{\mathrm{p}}\left(\varphi_{\mathrm{B}}\right)$;

(2) $\varphi_{\mathrm{B}}$ and $R_{\mathrm{s}}\left(\varphi_{\mathrm{B}}\right)$;

(3) $\varphi_{\mathrm{B}}$ and $R_{\mathrm{p}}\left(\varphi_{\mathrm{B}}\right) / R_{\mathrm{s}}\left(\varphi_{\mathrm{B}}\right)[53,54]$;

(4) $\varphi_{\mathrm{B}}$ and $R_{\mathrm{s}}, R_{\mathrm{p}}$, or $R_{\mathrm{p}} / R_{\mathrm{s}}$ at any $\varphi$;

(5) $R(0)$ and [d $\left.\ln R_{\mathrm{s}} / \mathrm{d} \varphi-\mathrm{d} \ln R_{\mathrm{p}} / \mathrm{d} \varphi\right]$ at $\varphi>0$ [55] ;

(6) $\mathrm{d} R_{\mathrm{p}} / \mathrm{d} \varphi$ at two values of $\varphi$, both near $\varphi_{\mathrm{B}}$ [56];

(7) the maximum value of $\left(R_{\mathrm{s}}-R_{\mathrm{p}}\right) /\left(R_{\mathrm{s}}+R_{\mathrm{p}}\right)$ and the angle $\varphi$ which it occurs [57].

Some of these have not been used much in practice. Plots similar to that of figure 2 can be carried out from the Fresnel equations to demonstrate the sensitivity, or lack thereof, of these methods [51]. (For metals in the infrared, $\varphi_{\mathrm{B}}$ becomes large, $>70^{\circ}$ or $80^{\circ}$.) Determining the angle $\varphi_{\mathrm{B}}$ may be particularly difficult, for the noise in the detector or the photon beam becomes important as the reflected intensity becomes small (but $R_{\mathrm{p}}$ will not $\rightarrow 0$ for an absorbing sample) and the angular spread of the incident beam will be important, especially if the photocathode is inhomogeneous.

3.4 Measure the reflectance over a large frequency range and use Kramers-Kronig integrals to obtain the phase $\theta$ [58]. From these two quantities $\tilde{\varepsilon}$ can be determined. Any angle of incidence can be used if the beam is linearly polarized $[59,60]$. A great deal of discussion has taken place about this method because of the need for extrapolating data beyond the finite range in which data, one's own, or literature data, are available. The 
use of synchrotron radiation has diminished the range over which an extrapolation is needed [61]. Many methods have been proposed [62], and the sum rules may be used to check on consistency, but some people are still uneasy about getting something for nothing. By using a variety of extrapolations, one can easily determine how large the errors in a given spectral region may be. They can be very large indeed near the limits of the measured data, and quite small in the center of the range of measurement. If reliable $n$ and $k$ values exist at one frequency (from other measurements), the phase can be adjusted to force the KK method to give the known results [63, 64]. This can give very accurate values of $\tilde{\varepsilon}$ over a large range about this one frequency. One can generate reflectance data from a harmonic oscillator model, truncate the data range, and follow the errors introduced as the "data " range becomes smaller, the extrapolations are changed, or the phase is forced to fit the exact value at one or more points [64]. The errors in $\tilde{\varepsilon}$ due to errors in the measurement of $R$ are difficult to discuss. Errors in $R$ lead to errors in the phase. (A simple multiplication of $R$ by a constant factor does not change the phase, due to the form of the integral on $\ln R$ ). However, to get $n$ and $k$ from $R$ and $\theta$, one must use $R, \sqrt{R}, \sin \theta$, and $\cos \theta$, so errors must be assessed numerically.

Most practitioners of Kramers-Kronig techniques are more interested in the shape of the spectrum than in the accuracy of the magnitudes of $n$ and $k$. The biggest effect on $\tilde{\varepsilon}$ of an incorrect extrapolation of $R$ is to increase or decrease the magnitudes of $\varepsilon_{1}$ and $\varepsilon_{2}$, the peak positions remaining about the same and relative peak heights not changing much. Thus it is possible that the reflectance be measured with an accuracy of a percent or two, the extrapolations not be done well, and the author still be happy with the resultant spectra, which may be inaccurate by as much as 20 percent or more, because the spectral features are right. Such spectra may be adequate for much of the interpretation in terms of the electronic properties of the material, but they cannot be used reliably for assistance in other measurements.

Another caveat about the Kramers-Kronig technique is this. The infrared reflectance of a metal is high and difficult to measure accurately. Often the extrapolation is made to zero frequency by fitting to a Drude expression. This gives an electron relaxation time which is not very accurate. Large changes in this parameter lead to small changes in the refiectance in the infrared [65]. Moreover, part of the Drude fitting will usually be to interband absorption which is unresolved from the Drude background, and of unknown strength. As an extrapolation procedure, this is probably not so bad, but one must not use the Drude parameters so obtained for other purposes, either to be interpreted themselves or to produce a Drude term at higher frequencies for subtraction from the measured $\tilde{\varepsilon}$ to reveal the "pure" intraband contribution to $\tilde{\varepsilon}$.
A variation of the Kramers-Kronig method is to measure the reflectance over as wide a range as possible then fit it with the reflectance of a series of harmonic oscillators [66]. These oscillators themselves need have no physical significance, but the $\tilde{\varepsilon}$ they generate represents accurately that of the material under study. This method has been tested on data from several types of solids and seems to work very well.

All of the foregoing methods are usually used with bulk samples or with opaque film samples, although in many cases semitransparent thin films could be used with suitable modification of the equations. For anisotropic samples, single crystals must be used if the anisotropy is to be determined [67-71]. There are other methods which must be used on films alone, either because of a geometrical restriction or because at least one measurement is of the radiation transmitted through a semitransparent film. Some of these thin film measurements follow.

3.5 If $n$ is less than refractive index of a transparent substrate and $k \ll 1$, a plot of measured s-polarized reflectance (from the substrate side) versus $\varphi$ will exhibit a sharp rise as $\varphi$ increases to the critical angle for total internal reflectance. The entire plot can be fitted to obtain $n$ and $k$. If $n<1$, the radiation can be incident from vacuum. This method has been used on a number of metals in the vacuum ultraviolet [72, 73].

3.6 Measure $R$ and $T$ of a film of known thickness $d$ [74-86]. However, whenever $n \approx k$, the sensitivity to small errors in $R$ and $T$ is very large [77, 78,81]. One can then use radiation incident from the substrate side, but this usually only reduces, not eliminates, the frequency region in which the accuracy is poor. Multiple solutions for $n$ and $k$ may also occur. One also can treat $\mathrm{d}$ as an unknown and make three measurements, e. g. $T\left(0^{\circ}\right), R\left(0^{\circ}\right)$, and $T_{\mathrm{p}}(\varphi)$ with $\varphi \gtrsim 60^{\circ}$. The data at each frequency should yield the same value of $d$ [85].

\section{$3.7 T_{\mathrm{s}}(\varphi)$ and $T_{\mathrm{p}}(\varphi)$.}

$3.8 T\left(0^{\circ}\right)$ for two known thicknesses.

Many measurements that can be made on films have been discussed by Hansen [86].

3.9 One can Kramer-Kronig analyze the transmission of a film, or fit a set of oscillators to it, the oscillators then generating the dielectric function [87].

\subsection{Ellipsometry on a semitransparent film.}

3.11 Attenuated total reflectance spectroscopy [88] has been used to obtain optical constants. A film is evaporated on the bottom of a prism or hemicylinder. The angle of incidence of the p-polarized measuring beam is varied as in method (6). If the film is not very thick, the fields penetrate the film to the lower surface, and air-metal (or vacuum) interface, at which a surface plasmon may be excited. (Because of the strong coupling between this plasmon and the photon, the excited mode is called a surface polariton.) This produces a dip in the spectrum of reflectance versus angle 
of incidence, for the reflectance is far less than total as energy is removed from the photon beam by the (nonradiative) surface plasmons. Since these plasmons are excited by the evanescent waves at the interface, they need not be excited at only a single plasma frequency. Consequently, one can determine the optical constants of the metal film by fitting the measured reflectance versus angle curves over a considerable range of frequencies below the plasma frequency by this method [89]. One can also carry out ellipsometric measurements on the reflected beam [90].

3.12 Dielectric functions can be measured by a nonoptical technique, the measurement of the spectrum of energy losses suffered by a fast electron as it traverses a thin film sample. The probability that a fast electron of energy $E_{0}(\gtrsim 1 \mathrm{keV})$ will lose energy between $E$ and $E+d E$ in a single inelastic scattering event into $\mathrm{d} \theta$ at scattering angle $\theta$ in passing through a film of thickness $d$ is

$$
\frac{1}{\pi} \frac{d}{a_{0}} \frac{\mathrm{d} E}{E_{0}} \operatorname{Im}\left(\frac{-1}{\tilde{\varepsilon}(E)}\right) \frac{\theta \mathrm{d} \theta}{\theta^{2}+\theta_{\mathrm{E}}^{2}}
$$

in which $a_{0}$ is the first Bohr radius and $\theta_{\mathrm{E}}=E /\left(2 E_{0}\right)$. This probability can be measured $[91,92]$. The measured spectrum also contains the effects of scattering at the surface and of multiple scattering in the bulk. These can be determined by measuring the spectra for several values of $d$ and $\theta$. The resultant corrected spectrum then gives $\operatorname{Im}(-1 / \tilde{\varepsilon})$. This can be subjected to a Kramers-Kronig analysis to obtain $\operatorname{Re}(-1 / \tilde{\varepsilon})$, from which $\tilde{\varepsilon}$ itself can be obtained. This method can be improved by using sum rules. It is also possible to obtain a spectrum proportional to $\operatorname{Im}(-1 / \tilde{\varepsilon})$ by using electrons backscattered from a bulk sample, but the actual magnitudes cannot be obtained this way. Electron energy loss measurements have been used to obtain the $\tilde{\boldsymbol{\varepsilon}}$ spectrum of a number solids up to an energy of the order of $100 \mathrm{eV}[93,94]$. Data below about $1 \mathrm{eV}$ are normally rather difficult to obtain reliably by this method, however, because of overlap of the inelastic spectrum with the tail of the elastically scattered beam. (This can be circumvented.) Electron energy loss spectroscopy can be used to obtain the components of the dielectric function tensor for anisotropic materials if sufficiently thin single crystalss can be obtained [95-98].

It is not difficult to calculate the error in $n$ and $k$ from errors in the measured quantities, but the errors in the latter are often very difficult to estimate. Assuming even this can be done, we are left with the most diffcult part of error analysis - how much does the sample we are measuring resemble the ideal sample used with Maxwell's equations and the ideal sample with whose electronic structure we might want to compare the resultant $\tilde{\varepsilon}$ ? With bulk samples, sawing or spark cutting can produce strains as deep as $50 \mu \mathrm{m}$ below the surface (even more for some types of sawing). After polishing, the surface layer may be very strained.
Electropolishing can remove a great deal of strained material without introducing more strain, but it may leave a surface that is less flat than the original polished one, one which is covered with a protective film. The surface layer left after electropolishing will affect some types of measurements, e. g., ellipsometry, more than others, e. g., a reflectance measurement, although even in the latter case the errors will be larger than we have indicated previously near the plasma minimum or in spectral regions in which the film is absorbing. Moreover, not all metals, e. g. Pt, can be electropolished. Annealing also can remove surface strains, but for metals this may accelerate oxidation of the surface if not carried out in ultrahigh vacuum, and, in many cases, it will leave the surface roughened. Surface roughness will remove radiation from the reflected beam, causing errors in almost all photometric measurements, and, since scattering is polarization-sensitive, it will cause errors in ellipsometric measurements as well.

Evaporated films are notoriously strained, and this can lead to broadened spectral features. Small-grained films have grain boundaries which contribute to optical properties [99-101]. Films may be annealed, but at the expense of increased surface roughness. Moreover, the annealing may be less effective than for bulk samples if there is bonding between the film and a substrate of different expansion coefficient. Unsupported films, presumably with less strain than in supported films, may be used for electron energy loss measurements, or for transmission measurements, but their surfaces are usually too poor to allow reflectance or ellipsometric measurements. It often is difficult to obtain and keep clean films of reactive substances. The evaporating material may getter residual oxygen and, if the films must be removed from the vacuum chamber for measurement, the atmosphere often will oxidize an evaporated film more rapidly than a bulk sample. The films may be protected by an inert, transparent overlayer, but it is difficult to obtain optical properties of films in the visible with such a film even when its characteristics are well known [102]. (But see ref. [102'].)

4. Some examples. - We close by illustrating several sets of measurements on some common materials. The agreement among different investigators often is poorer than the published errors lead one to believe. Rather than assuming the quoted errors are too small, one should look for differences in samples. No attempt is made to include all measurements on the metals used as illustration, nor is a selection made of the best data from among conflicting measurements.

Mercury has been the metal of most interest in electrochemistry to date. The long history of optical measurements and the disagreement among the results of some of the most recent and most carefully done measurements has been surveyed by Bloch and Rice [103]. They have used a model for the surface in which the charge density varies from the bulk value 
to zero in such a way that their reflectance data and the ellipsometric data of Smith [104], seemingly in conflict, can be reconciled.

Figure 3 shows $\varepsilon_{2}$ for $\mathrm{Al}$ in the near infrared and visible. The first three curves are from attenuated total

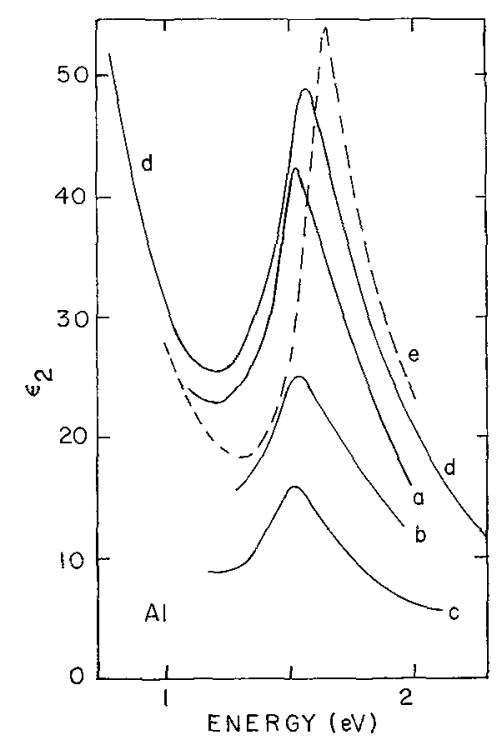

FIG. 3. - Imaginary part of the dielectric function for Al. Curves $a, b$, and $c$ are from reference [105] and were obtained by. attenuated total reflectance on glass, $\mathrm{MgF}_{2}$, and $\mathrm{LiF}$ substrates, respectively. Curves $\mathrm{d}$ and $\mathrm{e}$ are from references [107] and [108]. See text.

reflectance measurements using the same apparatus and sample preparation technique, except for the use of different substrates for condensing the Al films [105]. The differences in the spectra evidently are the result of different degrees of structural disorder in the films, which appears to depend on the substrate material and its microscopic surface configuration. The peak in $\varepsilon_{2}$ is the result of interband transitions between bands that are nearly parallel. It is known to diminish to near insignificance in highly disordered films [106], but the spectra in figure 3 are sharper and more prominent than in such films. The other two spectra were obtained by ellipsometry [107] on an evaporated film and by reflectance (actually absorptance) measurements at $4 \mathrm{~K}$ on a bulk sample, followed by Kramers-Kronig analysis [108]. The increased height of the peak and its shift to higher energy is largely a result of the lower temperature.

The use of thin films does not always lead to such problems. Théye has made measurements on very thin films of $\mathrm{Au}$ [109]. By evaporating films only a few hundred $\AA$ thick and annealing them so that the grains were between 3000 and $5000 \AA$ in diameter, she obtained reproducible results in agreement with measurements on bulk samples. Too little annealing or too much annealing produced different results, and films evaporated onto heated substrates gave rise to an anomalous absorption below the first absorption edge, previously noticed by others, but not known to be spurious. This was attributed to defects, or impurities which did not anneal out because the films evaporated on hot substrates did not experience subsequent grain growth upon further annealing.

Figure 4 shows $\varepsilon_{2}$ for $\mathrm{Cu}$ in the visible and near ultraviolet. Curve a was obtained by ellipsometry on

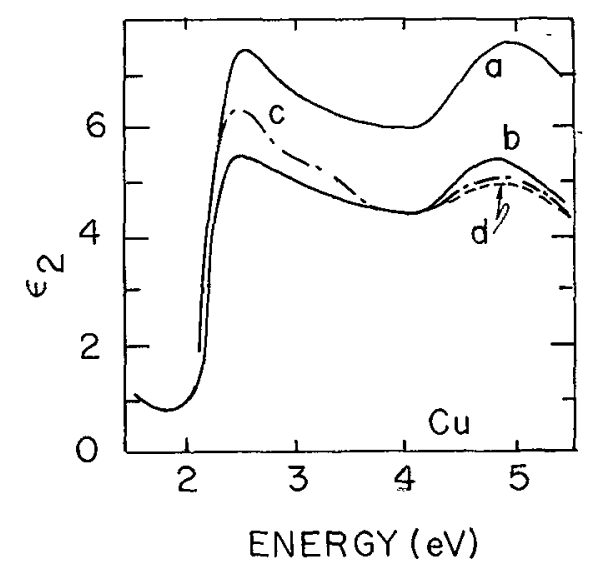

Fig. 4. - Imaginary part of the dielectric function of $\mathrm{Cu}$ Curves $a, b, c$ and $d$ are from reference [110-113], respectively. See text for further description.

annealed bulk samples [110]. Curve b was obtained by Kramers-Kronig analysis of the reflectance of a single crystal [111]. Curve $c$ came from measurements of reflectance and transmittance of thin films [112] and d is from measurements similar to those of curve $b$ [113]. Not shown clearly in this figure are several small structures, changes in slopes, that are manifest in some, but not all, of the spectra.

Figure 5 shows $\varepsilon_{2}$ for $\mathrm{Ni}$, but with the wavelength as the abscissa. The top two $[114,115]$ and bottom two $[118,119]$ curves were obtained ellipsometrically. The others are from the reflectance and transmittance of thin films [116] and from reflectance measurements

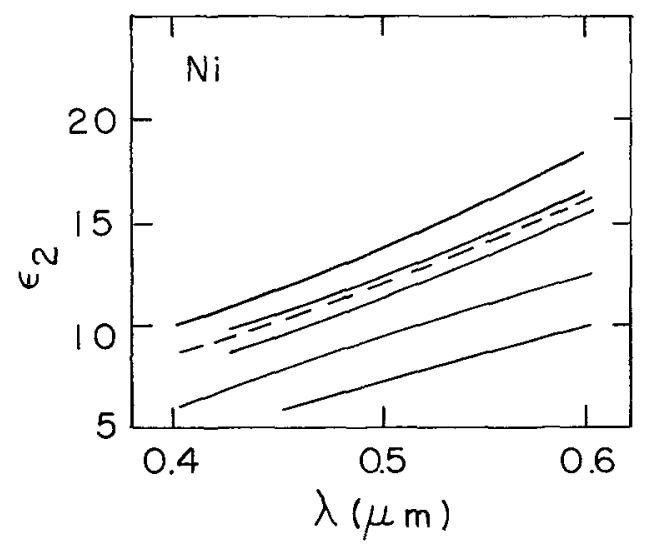

FIG. 5. - Imaginary part of the dielectric function of Ni. The curves from top to bottom are from references [114-119], respectively. (From Ref. [120].) See text. 
on a single crystal [117]. Most of the ellipsometric work was on bulk or single crystal samples. Again, several of the original spectra show fine structure in

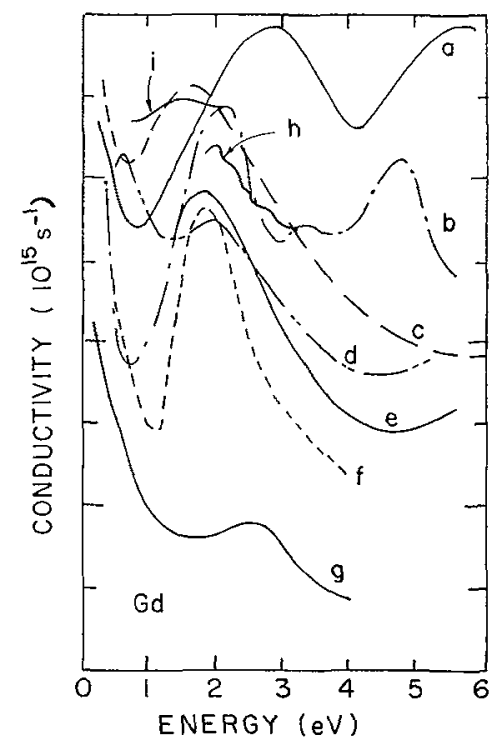

FIG. 6. - Real part of the conductivity of Gd. Curves a-i are from references [121-129], respectively. See text. this region, but this structure has not been obtained in other spectra made in an attempt to confirm it.

Figure 6 shows the conductivity resulting from many measurements on $\mathrm{Gd}$, a very reactive metal compared with the previous examples. Curves $d, e$, and $f$ agree fairly well, $d$ and e having been obtained ellipsometrically on films (annealed films in the case of $d$, for unannealed films gave different spectra) while KramersKronig analysis of reflectance (or absorptance) data on single crystals was used for curve $f$. Most of the other curves came from data on films. The spread in the magnitude of the conductivity at any one energy is beyond the estimated errors of the data, based on measurement errors, and is due to difference in samples.

5. Summary. - The experimental evaluation of the dielectric function for a metallic sample is a difficult task. A variety of methods are available, all of which can be made to agree to within a few percent if the samples can be controlled.

Acknowledgments. - This work was supported by the U. S. Energy Research and Development Administration, Division of Physical Research.

References

[1] STern, F., Solid State Phys. 15 (1963) 299.

[2] Wooten, F., Optical Properties of Solids (Academic Press, New York) 1972.

[3] Sokolov, A. V., Optical Properties of Metals (Blackie, London) 1967.

[4] NYE, J. F., Physical Properties of Crystals (Oxford Univ. Press, London) 1957, Sect. 5.1.

[5] Jones, H., Handb. Phys. 19 (1956) 308.

[6] PrPpard, A. B., Dynamics of Conduction Electrons (Gordon and Breach, New York) 1965, p. $58 \mathrm{ff}$.

[7] Bennett, H. E., Bennett, J. M., Ashley, E. J. and MotYKa, R. J., Phys. Rev. 165 (1968) 755.

[8] KuIEwer, K. L. and Fuchs, R., Phys. Rev. 172 (1968) 607.

[9] Kliewer, K. L. and Fuchs, R., Phys. Rev. B 2 (1970) 2923.

[10] McInTyre, J. D. E. and AsPNes, D. E., Surf. Sci. 24 (1971) 417.

[11] MCINTYRe, J. D. E. in Seraphin, B. O., ed., Optical Properties of Solids New Developments (North-Holland, Amsterdam) 1976, p. 555.

[12] Del Sole, R., J. Phys. C 8 (1975) 2971.

[13] Feibelman, P. J., Phys. Rev. B 12 (1975) 1319.

[14] Feibelman, P. J., Phys. Rev. B 14 (1976) 762.

[15] KLIEWER, K. L. in Feuerbacher, B., Fitton, B. and Willis, R. F., eds., Photoemission from Surfaces (John Wiley and Sons, New York) 1977, ch. 3.

[16] Altarelli, M., DeXter, D. L., Nussenzveig, H. M., Smith, D. Y., Phys. Rev. B 6 (1972) 4502.

[17] Villani, A. and Zrmmerman, A. H., Phys. Rev. B 8 (1973) 3914.

[18] Altarelli, M. and Smith, D. Y., Phys. Rev. B9 (1974) 1290

[19] Wolter, H., Hand. Phys. 24 (1956) 461.

[20] GoedeCKE, G. H., J. Opt. Soc. Am. 65 (1975) 146.

[21] KRöGER, E., J. Opt. Soc. Am. 65 (1975) 1075.

[22] Chambers, W. G., Infrared Phys. 15 (1975) 139.

[23] Sмiтr, D. Y., J. Opt. Soc. Am. 67 (1977) 570.

[24] Young, R. H., J. Opt. Soc. Am. 67 (1977) 520.

[25] Fromhold, A. T., Nuovo Cimento 28 (1963) 1127.
[26] Bennett, H. E. and Porteus, J. O., J. Opt. Soc. Am. 51 (1961) 123.

[27] Porteus, J. O., J. Oft. Soc. Am. 53 (1963) 1394

[28] BennetT, H. E., J. Opt. Soc. Am. 53 (1963) 1389.

[29] Berreman, D. W., Phys. Rev. 163 (1967) 855.

[30] Berreman, D. W., Phys. Rev. B 2 (1970) 381.

[31] Berreman, D. W., J. Opt. Soc. Am. 60 (1970) 499.

[32] Christy, R. W., Am. J. Phys. 40 (1972) 1403.

[33] Haga, E. and Aisaka, T., J. Phys. Soc. Japan 22 (1967) 987

[34] Nilsson, P. O., Solid State Phys. 29 (1974) 139.

[35] AsPnes, D. E., in Seraphin, B. O., ed., Optical Properties of Solids New Developments (North-Holland, Amsterdam) 1976, p. 799.

[36] Schledermann, M. and Skibowski, M., Appl. Opt. 10 (1971) 321.

[37] JASPerson, S. N. and Schnatterly, S. E., Rev. Sci. Instrum. 40 (1969) 761.

[38] KeMP, J. C., J. Opt. Soc. Am. 59 (1969) 950.

[39] Mollenauer, L. F., Downie, D., Engstrom, H. and Grant, W. B., Appl. Opt. 8 (1969) 661.

[40] Palmer, R. E. and Schnatterly, S. E., Phys. Rev, B 4 (1971) 2329.

[41] Treu, J. I., Callender, A. B. and Schnatteriy, S. E., Rev. Sci. Instrum. 44 (1973) 793.

[42] O'Handley, R. C., J. Opt. Soc. Am. 63 (1973) 523.

[43] Simon, I., J. Opt. Soc. Am. 41 (1951) 334.

[44] Hunter, W. R., J. Opt. Soc. Am. 55 (1965) 1197.

[45] Hunter, W. R., Appl. Opt. 6 (1967) 2140.

[46] Miller, R. E., TAYLoR, A. J. and Julien, L. S., J. Phys. D 3 (1970) 1957.

[47] ARMaly, B. F., OchOA, J. G. and Look, D. C., Appl. Opt. 11 (1972) 2907.

[48] Avery, D. G., Proc. Phys. Soc. B 65 (1952) 425.

[49] Lindquist, R. E. and Ewald, A. W., J. Opt. Soc. Am. 53 (1963) 247.

[50] Field, G. R. and Murphy, E., Appl. Opt. 10 (1971) 1402.

[51] Humphrey-Owen, S. P. F., Proc. Phys. Soc. 77 (1961) 949. 
[52] BennetT, H. E. and Bennett, J. M., in Hass, G. and Thun, R.E., eds., Physics of Thin Films (Academic Press, New York) 1967, Vol. 4, p. 1.

[53] Potter, R. F., J. Opt. Soc. Am. 54 (1964) 904.

[54] PotTer, R. F., Appl. Opt. 4 (1965) 53.

[55] Hunderi, O., Appl. Opt. 11 (1972) 1572.

[56] Balzarotti, A., Ptcozzi, P., and Santucci, S., Surf. Sci. 37 (1973) 994.

[57] SCHMIDT, E., Appl. Opt. 8 (1969) 1905.

[58] Robrnson, T. S., Proc. Phys. Soc. B 65 (1952) 910.

[59] Roessler, D. M., Br. J. Appl. Phys, 16 (1965) 1359.

[60] Berreman, D. W., Appl. Opt. 6 (1967) 1519.

[61] LYNCH, D. W., in Kunz, C., ed., Synchrotron Radiation I., Spring Topics in Current Series, to be published.

[62] Veal, B. W. and Paulikas, A. P., Phys. Rev. B 10 (1974) 1280.

[63] Roessier, D. M., Br. J. Appl. Phys. 17 (1966) 1313.

[64] Nilsson, P. O. and Munkby, L., Phys. Kond. Mater. 10 (1969) 290.

[65] Wooten, F., Pajanne, E. and Bergersen, B., Phys. Status Solidi 37 (1970) 367.

[66] Verleur, H. W., J. Opt. Soc. Am. 58 (1968) 1356.

[67] UzAN, E., Opt. Acta 15 (1968) 237.

[68] Mosteller, L. P. and Wooten, F., J. Opt. Soc. Am. 58 (1968) 511.

[69] Graves, R. W. H. and Lenham, A. P., J. Opt. Soc. Am. 58 (1968) 884.

[70] Berman, M., Kerchner, H. R. and Ergun, S., J. Opt. Soc. Am. 60 (1970) 646.

[71] Koch, E. E., Otro, A. and Kutewer, K. L., Chem. Phys. 3 (1974) 362.

[72] HunTER, W. R., J. Opt. Soc. Am. 54 (1964) 15.

[73] Whang, U. S., Arakawa, E. T. and Callcott, T. A., J. Opt. Sac. Am. 61 (1971) 740.

[74] Heavens, O. S., Optical Properties of Thin Solid Films (Butterworths, London) 1950.

[75] Abelés, F., in Wolf, E., ed., Progress in Optics, Vol. II (1963) p. 249.

[76] VASICEK, A., Optics of Thin Films (North-Holland, Amsterdam) 1960.

[77] Abelés, F. and ThéYe, M. L., Surf. Sci. 5 (1966) 325.

[77'] BENNETT, J. M. and Booty, M. J., Appl. Opt. 5 (1966) 41.

[78] Grant, P. M. and Paul, W., J. Appl. Phys. 37 (1966) 3110.

[79] WARD, L. and NAG, A., Br. J. Appl. Phys. 18 (1967) 277.

[80] Ward, L. and NaG, A., Br. J. Appl. Phys. 18 (1967) 1629.

[81] Nir.sson, P. O., Appl. Opt. 7 (1968) 435.

[82] Ward, L. and Nag, A., J. Phys. D 3 (1970) 462.

[83] BALdin,, G. and Rigald, L., J. Opt. Soc. Am. 60 (1970) 495.

[84] Nesteld, J. E. and Christy, R. W., Am. J. Phys. 39 (1971) 313.

[85] Nestell, J. E. and Christy, R. W., Appl. Opt. 11 (1972) 643.

[86] Hansen, W. N., J. Opt. Am. 63 (1973) 793.

[87] Rivory, J., Opt. Commun. 1 (1970) 334.

[88] Oтто, A., in Seraphin, B. O., ed., Optical Properties of Solids New Developments (North-Holland, Amsterdam) 1976, p. 677.

[89] Kretschmann, E., Z. Phys. 241 (1971) 313.

[90] ABelés, F. and Lopez-Rros, T., Opt. Commun. 11 (1974) 89.
[91] Raether, H., Springer Tracts Mod. Phys. 38 (1965) 84. [92] Daniels, J., Festenberg, C. V., RakTHer, H. and ZePPenFELD, K., Springer Tracts Mod. Phys. 54 (1970) 77.

[93] KeIL, P., Z. Phys. 214 (1968) 251, 266.

[94] Daniels, J., Z. Phys. 227 (1969) 234.

[95] ZePPENFELd, K., Z. Phys. 211 (1968) 391.

[96] Tosatti, E., Nuovo Cimento 63B (1969) 54 ; 65B (1970) 280.

[97] Venghaus, H., Z. Phys. 239 (1970) 289.

[98] Tosatti, E. and Bassant, F., Nuovo Cimento 65B (1970) 161.

[99] Hunderi, O., Solid State Commun. 12 (1973) 237.

[100] Nagel, S. R. and Schnatterly, S. E., Phys, Rev. B 9 (1974) 1299.

[101] Nagel, S. R. and Schnatterly, S. E., Phys. Rev. B 12 (1975) 6002.

[102'] Bennetr, J. M., Ashley, E. J. and Bennett, H. E., Appl. Opt. 4 (1965) 961.

[102] Blondeau, G., Froblicher, M., Froment, M. and HugotLegoff, A., Thin Solid Films 38 (1976) 261.

[103] Bloch, A. N. and Rice, S. A., Phys. Rev. 185 (1969) 933.

[104] Sмith, N. V., Adv. Phys. 16 (1967) 629.

[105] Oтto, A. and Sohler, W., Solid State Commun. 16 (1975) 1319.

[106] Bernland, L., Hunderi, O. and Myers, H. P., Phys. Rev. Lett. 31 (1975) 363.

[107] Mathewson, A. G. and Myers, H. P., Phys. Scripta 4 (1971) 291.

[108] Benbow, R. L. and LynCH, D. W., Phys. Rev. B 12 (1975) 5615 .

[109] ThÉYe, M. L., Phys. Rev. B 2 (1970) 3060.

[110] Peiss, G. P. and Shrga, M., J. Phys. C 2 (1969) 1835.

[111] Grrhardt, U., Phys. Rev. 172 (1968) 651.

[112] Jomnson, P. B. and Christy, R. W., Phys. Rev. B 11 (1975) 1315.

[113] Beaglehole, D., Proc. Phys. Soc. 85 (1965) 1007.

[114] Shiga, H. and Pelis, G. P., J. Phys. C 2 (1969) 1847.

[115] Stoll, M. P., Solid State Commun. 8 (1970) 1207.

[116] Johnson, P. B. and Christy, R. W., Phys. Rev. B 9 (1974) 5056 .

[117] LyNCh, D. W., Roser, R. and Weaver, J. H., Solid State Commun. 9 (1971) 2195.

[118] KirLlova, M. M., Sov. Phys. JETP 34 (1972) 178.

[119] Studna, A. A., Solid State Commun. 16 (1975) 1063.

[120] Veal, B. W. and Paulikas, A. P., Int. J. Magni. 4 (1973) 57.

[121] Petrakian, J. P., Thin Solid Films 13 (1972) 269 ; C. R. Hebd. Séan. Acad. Sci. B $270 ; J$. Opt. Soc. Am. 62 (1970) $401 ; 1760$ (624).

[122] SChüler, C. Ch., in Abelés, F., ed., Optical Properties and Electronic Structure of Metals and Alloys (NorthHolland, Amsterdam) 1966, p. 221.

[123] Hodgson, J. N. and Cleyet, V., J. Phys. C 2 (1969) 97.

[124] Myers, H. P., J. Phys. F 6 (1976) 141.

[125] Erskine, J. L., Blake, G. A. and Flaten, C. J., J. Opt. Soc. Am. 64 (1974) 1332.

[126] Weaver, J. H. and LyNCH, D. W., Phys. Rev, Lett. 34 (1975) 1324.

[127] KNyAZev, Y. V., Fiz. Met. Metalloved. 30 (1970) 214 ; 31 (1971) 1099 ; 32 (1971) 1189.

[128] Mrller, R. F., Julien, L. S., and TAYlor, A. J., J. Phys. F $4(1974) 2338$.

[129] KrizeK, J. and TaYloR, K. N. R., J. Phys. F 5 (1975) 774. 